\title{
LEVEL CROSSING ORDERING OF SKIP-FREE-TO-THE-RIGHT CONTINUOUS-TIME MARKOV CHAINS
}

\author{
FÁTIMA FERREIRA,* CEMAT and Universidade de Trás os Montes e Alto Douro \\ ANTÓNIO PACHECO, ${ }^{* *}$ CEMAT and Instituto Superior Técnico, Universidade Técnica de Lisboa
}

\begin{abstract}
As proposed by Irle and Gani in 2001, a process $X$ is said to be slower in level crossing than a process $Y$ if it takes $X$ stochastically longer to exceed any given level than it does $Y$. In this paper, we extend a result of Irle (2003), relative to the level crossing ordering of uniformizable skip-free-to-the-right continuous-time Markov chains, to derive a new set of sufficient conditions for the level crossing ordering of these processes. We apply our findings to birth-death processes with and without catastrophes, and $\mathrm{M} / \mathrm{M} / s / c$ systems.
\end{abstract}

Keywords: Birth-death process with catastrophe; continuous-time Markov chain; level crossing ordering; $\mathrm{M} / \mathrm{M} / s / c$ system; stochastic ordering

2000 Mathematics Subject Classification: Primary 60E15; 60J27

Secondary 60J80; 60K 25

\section{Introduction}

In this paper, we consider the level crossing ordering of stochastic processes proposed by Irle and Gani [6]. A process $X$ is said to be slower in level crossing than a process $Y$ if it takes $X$ stochastically longer to exceed any given level than it does $Y$, with the random variables being compared through the usual stochastic order (in distribution).

Irle and Gani [6] showed that the ordering in distribution of the transition probabilities of skip-free-to-the-right discrete-time Markov chains (DTMCs) for any common initial state (which does not guarantee the usual stochastic ordering of the respective DTMCs) implies the level crossing ordering of the DTMCs. Later, the analysis was extended (see [5]) to semiMarkov processes (SMPs) and a set of sufficient conditions for the level crossing ordering of skip-free-to-the-right SMPs was derived (see [5, Theorem 2.1]), whose translation for skipfree-to-the-right continuous-time Markov chains (CTMCs) $X^{(1)}$ and $X^{(2)}$ is as follows:

(a) The transition probabilities of the embedded DTMCs (at transition epochs) associated to $X^{(1)}$ and $X^{(2)}$ are ordered, in distribution, for any common initial state.

(b) The transition rates from states are smaller in $X^{(1)}$ than in $X^{(2)}$.

Accordingly, we call these Irle's (set of sufficient) conditions for the level crossing ordering of skip-free-to-the-right CTMCs.

Received 4 May 2004; revision received 22 September 2004.

* Postal address: Departamento de Matemática, Universidade de Trás os Montes e Alto Douro, Quinta dos Prados, Apartado 1013, 5001-911 Vila Real, Portugal. Email address: mmferrei@utad.pt

** Postal address: Departamento de Matemática, Instituto Superior Técnico, Universidade Técnica de Lisboa, Av. Rovisco Pais, 1049-001 Lisboa, Portugal. Email address: apacheco@math.ist.utl.pt 
In this paper, we essentially show that if $X^{(1)}$ and $X^{(2)}$ are two uniformizable (see [10]) skipfree-to-the-right CTMCs with (infinitesimal) generator matrices $\boldsymbol{Q}^{(1)}$ and $\boldsymbol{Q}^{(2)}$, respectively, and if there exists a positive real constant $\alpha \in(0,1]$ such that

$$
\sum_{j \geq k} q_{i j}^{(1)} \leq \alpha \sum_{j \geq k} q_{i j}^{(2)} \quad \text { for all } i \text { and } k,
$$

where $q_{i j}^{(k)}, k=1,2$, are the entries of $\boldsymbol{Q}^{(k)}$, then $X^{(1)}$ is slower in level crossing than $X^{(2)}$. This result extends [5, Corollary 2.1], which established the same conclusion for $\alpha=1$. The set of conditions given by (1), with $\alpha=1$, constitutes a relaxation of Kirstein's [7] sufficient conditions, i.e.

$$
\sum_{j \geq k} q_{i j}^{(1)} \leq \sum_{j \geq k} q_{m j}^{(2)} \quad \text { for all } i \leq m \text { and } k \leq i \text { or } k>m,
$$

for $X^{(1)}$ to be stochastically smaller than $X^{(2)}$ in the usual sense if their initial distributions are ordered in the same way, where the CTMCs do not need to be either uniformizable or skip-free to the right.

The sufficient conditions (1) for the level crossing ordering of uniformizable skip-free-to-theright CTMCs may lead to positive conclusions in situations where some of Kirstein's conditions (2) and Irle's conditions ((a) and (b), above) do not hold. They constitute a relevant addition to Irle's conditions, to compare uniformizable skip-free-to-the-right CTMCs in the sense of level crossing ordering, and are easier to check than Irle's.

Following works related to the growth of populations subject to catastrophes (see, for example, [1], [2], [3], and references therein), we illustrate the use of (1) for the level crossing ordering of uniformizable skip-free-to-the-right CTMCs by comparing birth-death processes with catastrophes (BDC processes).

We end this introduction with a brief outline of the paper. In Section 2, we introduce a few definitions and provide some relevant preliminary results and notation. In Section 3, we establish the main result of the paper on the level crossing ordering of skip-free-to-the-right CTMCs and, in Section 4, illustrate its application to BDC processes and, in particular, birthdeath processes and $\mathrm{M} / \mathrm{M} / \mathrm{s} / \mathrm{c}$ systems.

\section{Preliminaries}

In this section, we provide the definition of level crossing ordering of stochastic processes, as proposed in [6], state an important property of this stochastic order relative to time-clock changes, and introduce some notation that is used throughout the paper.

We next introduce notation for the hitting time of a set by a stochastic process and recall the definition of the usual order (in distribution) for random variables, $\leq_{\mathrm{st}}$. These are used in the subsequent definition of level crossing ordering of stochastic processes. We let $\mathbb{N}=$ $\{0,1,2, \ldots\}, \mathbb{N}_{+}=\{1,2, \ldots\}, \mathbb{R}_{+}=[0,+\infty)$, and let $I$ denote a countable ordered set. If $I$ is order-isomorphic to a bounded or unbounded interval of $\mathbb{Z}$, we let $\bar{I}=I \backslash\{\sup I\}$. Moreover, we use the convention that inf $\varnothing=\infty$.

Given a stochastic process $X=(X(t))_{t \in \mathbb{R}_{+}}$, taking values in $I$, and any two states $i$ and $l$, we let $S_{i, l}^{X}$ denote the hitting time by $X$ of the set of values greater than or equal to $l$, when departing from state $i$, i.e.

$$
S_{i, l}^{X}=\left[\inf \left\{t \in \mathbb{R}_{+}: X(t) \geq l\right\} \mid X(0)=i\right] .
$$


We recall that if $Y$ and $Z$ are random variables taking values in $I$, then $Y$ is stochastically smaller than $Z$ in the usual sense, i.e. $Y \leq_{\text {st }} Z$, if $\mathrm{P}(Y \leq i) \geq \mathrm{P}(Z \leq i)$ for all $i \in I$ (see, for example, [9] and [11]). Similarly, if $\boldsymbol{p}=\left(p_{i}\right)_{i \in I}$ and $\boldsymbol{q}=\left(q_{i}\right)_{i \in I}$ are two probability vectors, then we write $\boldsymbol{p} \leq_{\mathrm{st}} \boldsymbol{q}$ if $\sum_{i \leq j} p_{i} \geq \sum_{i \leq j} q_{i}$ for all $j \in I$. Thus, if the random variables $Y$ and $Z$, taking values in $I$, have probability vectors $\boldsymbol{p}$ and $\boldsymbol{q}$, respectively, then $Y \leq_{\mathrm{st}} Z$ if and only if $\boldsymbol{p} \leq_{\text {st }} \boldsymbol{q}$.

Definition 1. Given two stochastic processes $Y=(Y(t))_{t \in \mathbb{R}_{+}}$and $Z=(Z(t))_{t \in \mathbb{R}_{+}}$with common ordered state space $I$, the process $Y$ is said to be (stochastically) slower in level crossing than $Z$, denoted by $Y \leq_{\mathrm{lc}} Z$, if $S_{i, l}^{Z} \leq_{\mathrm{st}} S_{i, l}^{Y}$ for all $i, l \in I$.

We next introduce the definition of a time-clock speed change of a stochastic process, followed by the result that stochastic processes are stochastically monotone increasing, in the sense of level crossing ordering, with respect to time-clock speed.

Definition 2. Given a stochastic process $X=(X(t))_{t \in \mathbb{R}_{+}}$and a positive real constant $\alpha$, we let $X_{\alpha}=\left(X_{\alpha}(t)\right)_{t \in \mathbb{R}_{+}}$, where $X_{\alpha}(t)=X(\alpha t)$ for $t \in \mathbb{R}_{+}$, denote the time-clock speed change of $X$ by a factor of $\alpha$.

If $0<\alpha<1$ then $X_{\alpha}$ is a time dilation of $X$, and if $\alpha>1$ then $X_{\alpha}$ is a time contraction of $X$. Note that $X_{1}=X$ and $\left(X_{\alpha}\right)_{\beta}=\left(X_{\beta}\right)_{\alpha}=X_{\alpha \beta}$, for all $\alpha, \beta>0$, meaning that the time-clock speed change operation is both associative and commutative.

Theorem 1. Given a stochastic process $X=(X(t))_{t \in \mathbb{R}_{+}}$with ordered state space, the $\alpha$-parametrized family of processes $\left\{X_{\alpha}, \alpha>0\right\}$ is stochastically increasing in the sense of level crossing ordering, i.e. $X_{\alpha_{1}} \leq_{\mathrm{lc}} X_{\alpha_{2}}$ for all $\alpha_{1} \leq \alpha_{2}$.

Proof. Let $X=(X(t))_{t \in \mathbb{R}_{+}}$be a stochastic process with ordered state space $I$. From the definition of time-clock speed change, it follows that $X(t)=X_{\alpha}\left(\alpha^{-1} t\right)$, for all $\alpha>0$ and $t \in \mathbb{R}_{+}$, thus implying that $S_{i, l}^{X_{\alpha}}=\alpha^{-1} S_{i, l}^{X}$ for all $i, l \in I$ and $\alpha>0$. Thus, for $0<\alpha_{1} \leq \alpha_{2}$, $S_{i, l}^{X_{\alpha_{1}}}=\alpha_{1}{ }^{-1} S_{i, l}^{X} \geq \alpha_{2}{ }^{-1} S_{i, l}^{X}=S_{i, l}^{X_{\alpha_{2}}}$ for all $i, l \in I$. Therefore, $X_{\alpha_{1}} \leq_{\mathrm{lc}} X_{\alpha_{2}}$ as required.

In the paper, we address uniformizable and lower-uniformizable skip-free-to-the-right CTMCs. Following [10], we say that a CTMC $X$ with ordered state space $I$ and generator matrix $Q$ is uniformizable if $\sup _{i \in I} \sum_{j \neq i} q_{i j}<\infty$. Similarly, we say that $X$ is lower-uniformizable if $\sup _{i \leq l} \sum_{j \neq i} q_{i j}<\infty$ for all $l \in I$. In addition, if $I$ is order-isomorphic to some bounded or unbounded interval of $\mathbb{Z}$, then $X$ is said to be skip-free to the right if it almost surely does not have jumps up more than one level, i.e. $q_{i j}=0$ whenever $j>i+1$. Moreover, if $X$ has ordered state space, then $X$ is uniformizable, lower-uniformizable, or skip-free to the right if and only if the CTMC $X_{\alpha}$ is uniformizable, lower-uniformizable, or skip-free to the right, respectively.

We end the section with some notation and definitions. If $\boldsymbol{a}=\left(a_{i}\right)_{i \in I}$ and $\boldsymbol{b}=\left(b_{i}\right)_{i \in I}$ are two vectors, we say that $\boldsymbol{a} \leq \boldsymbol{b}$ if and only if $a_{i} \leq b_{i}$ for all $i \in I$. Moreover, given a stochastic matrix $\boldsymbol{A}=\left(a_{i k}\right)_{i, k \in I}$, we let $\boldsymbol{A}_{i}$. $=\left(a_{i j}\right)_{j \in I}$ denote the probability row vector containing row $i$ of $\boldsymbol{A}, i \in I$, and let $\underline{\boldsymbol{A}}=\left(\underline{a}_{i k}\right)_{i, k \in I}$ denote the matrix of the left-partial-row sums of $\boldsymbol{A}$, i.e. $\underline{a}_{i k}=\sum_{j \leq k} a_{i j}$. In addition, we let $\delta_{a b}$ denote the Kronecker delta function, i.e. $\delta_{a b}=1$ when $a=b$ and $\delta_{a b}=0$ otherwise. Finally, we let $\mathbf{1}_{A}$ denote the indicator function of statement $A$, i.e. $\mathbf{1}_{A}=1$ if $A$ is true and $\mathbf{1}_{A}=0$ otherwise. 


\section{Uniformizable skip-free-to-the-right continuous-time Markov chains}

Irle [5] investigated sufficient conditions for the level crossing ordering of uniformizable skip-free-to-the-right CTMCs and derived, in his Corollary 2.1, essentially the set of conditions (3) given in the next result, with the constant $\alpha$ taking the value 1.

Theorem 2. Let $X^{(1)}$ and $X^{(2)}$ be two lower-uniformizable skip-free-to-the-right CTMCs with common ordered state space I, order-isomorphic to some bounded or unbounded interval of $\mathbb{Z}$, with generator matrices $\boldsymbol{Q}^{(1)}$ and $\boldsymbol{Q}^{(2)}$, respectively.

Then, $X^{(1)} \leq_{1 \mathrm{c}} X^{(2)}$ provided that there exists a constant $\alpha \in(0,1]$ such that

$$
\sum_{j \geq k} q_{i j}^{(1)} \leq \alpha \sum_{j \geq k} q_{i j}^{(2)} \quad \text { for all } i \in \bar{I} \text { and } k \in I
$$

or, equivalently,

$$
\begin{aligned}
q_{i, i+1}^{(1)} & \leq \alpha q_{i, i+1}^{(2)} \quad \text { for all } i \in \bar{I}, \\
\sum_{j \leq k} q_{i j}^{(1)} & \geq \alpha \sum_{j \leq k} q_{i j}^{(2)} \quad \text { for all } i \in \bar{I} \text { and } k<i .
\end{aligned}
$$

Proof. Let $X^{(1)}$ and $X^{(2)}$ be as stated. Now, if $\alpha \in(0,1]$ denotes a constant such that (3) holds, and we let $\boldsymbol{Q}^{X_{\alpha}^{(2)}}=\alpha \boldsymbol{Q}^{(2)}$ denote the generator matrix of the CTMC $X_{\alpha}^{(2)}$, then, as $S_{\sup I, l}^{X^{(1)}}=S_{\sup I, l}^{X^{(2)}}=0$ for all $l \in I$, where sup $I<\infty$, and

$$
\sum_{j \geq k} q_{i j}^{(1)} \leq \alpha \sum_{j \geq k} q_{i j}^{(2)}=\sum_{j \geq k} q_{i j}^{X_{\alpha}^{(2)}} \quad \text { for all } i \in \bar{I} \text { and } k \in I,
$$

it follows that $X^{(1)} \leq_{\mathrm{lc}} X_{\alpha}^{(2)}$, in view of [5, Corollary 2.1]. Since, in addition, $\alpha \in(0,1]$, we conclude, using Theorem 1 , that $X^{(1)} \leq_{\mathrm{lc}} X_{\alpha}^{(2)} \leq_{\mathrm{lc}} X^{(2)}$. From the transitivity of the level crossing ordering, it then follows that $X^{(1)} \leq_{\mathrm{lc}} X^{(2)}$. As

$$
\sum_{j \geq k} q_{i j}^{(s)}=-\sum_{j<k} q_{i j}^{(s)} \mathbf{1}_{\{k \leq i\}}+q_{i, i+1}^{(s)} \delta_{k, i+1},
$$

the set of conditions ((4), (5)) is equivalent to (3), which concludes the proof.

Theorem 2 constitutes a simple but rich extension of [5, Corollary 2.1], as it greatly relaxes the sufficient conditions set forth in that result. An alternative proof of Theorem 2, which does not use Theorem 1, is based on an approach similar to the one used in the proof of [5, Corollary 2.1]. However, instead of uniformizing the processes $X^{(1)}$ and $X^{(2)}$ with the same uniformization rate, as done in [5], we would uniformize $X^{(1)}$ and $X^{(2)}$ with two possibly different uniformization rates $\alpha^{(1)}$ and $\alpha^{(2)}$, respectively, such that $\alpha=\alpha^{(1)} / \alpha^{(2)} \leq 1$.

We next give a result that provides sufficient conditions for the usual stochastic ordering of skip-free-to-the-right CTMCs. We recall that, given two random vectors $\boldsymbol{Y}=\left(Y_{1}, Y_{2}, \ldots, Y_{n}\right)$ and $\boldsymbol{Z}=\left(Z_{1}, Z_{2}, \ldots, Z_{n}\right)$, whose components take values in an ordered set $I$, the vector $\boldsymbol{Y}$ is smaller in the usual sense than $\boldsymbol{Z}$, written $\boldsymbol{Y} \leq_{\text {st }} \boldsymbol{Z}$, if $\mathrm{P}(\boldsymbol{Y} \in U) \leq \mathrm{P}(\boldsymbol{Z} \in U)$ for all upper sets $U$ of $I^{n}$. Note that $U$ is an upper set of $I^{n}$ if $\boldsymbol{x} \leq \boldsymbol{y}$, for some $\boldsymbol{x} \in U$, implies that $\boldsymbol{y} \in U$. Moreover, given two stochastic processes $Y=(Y(t))_{t \in \mathbb{R}_{+}}$and $Z=(Z(t))_{t \in \mathbb{R}_{+}}$with common ordered state space $I$, the process $Y$ is said to be strictly smaller (in the usual sense) than $Z$, denoted $Y \leq_{\text {st }} Z$, if for any $n \in \mathbb{N}_{+}$and $t_{1}, t_{2}, \ldots, t_{n} \in \mathbb{R}_{+},\left(Y\left(t_{k}\right)\right)_{k=1,2, \ldots, n} \leq_{\text {st }}\left(Z\left(t_{k}\right)\right)_{k=1,2, \ldots, n}$. See, for example, [8] and [11] for more details on the usual stochastic ordering. 
Lemma 1. Let $X^{(1)}$ and $X^{(2)}$ be two skip-free-to-the-right CTMCs with common ordered state space $I$, order-isomorphic to some bounded or unbounded interval of $\mathbb{Z}$, with generator matrices $\boldsymbol{Q}^{(1)}$ and $\boldsymbol{Q}^{(2)}$, respectively.

Then, $X^{(1)} \leq_{\mathrm{st}} X^{(2)}$ provided that $X^{(1)}(0) \leq_{\mathrm{st}} X^{(2)}(0)$ and

$$
\begin{aligned}
q_{i, i+1}^{(1)} & \leq q_{i, i+1}^{(2)} \quad \text { for all } i \in \bar{I}, \\
\sum_{j \leq k} q_{i j}^{(1)} & \geq \sum_{j \leq k} q_{m j}^{(2)} \quad \text { for all } k<i \leq m .
\end{aligned}
$$

Proof. For $s=1,2$, let $X^{(s)}$ be as stated and let $\boldsymbol{Q}^{(s)}$ denote the generator matrix of $X^{(s)}$. From, for example, [9, Theorem 5.2.19(a)] or [8, Theorem 6.29], it follows that if $X^{(1)}(0) \leq_{\text {st }} X^{(2)}(0)$ and the set of conditions (2) holds, then $X^{(1)} \leq_{\text {st }} X^{(2)}$. Thus, since the set of conditions ((7), (8)) is equivalent to (2) (from (6)), the result follows.

Although the usual stochastic ordering of CTMCs implies the corresponding level crossing ordering, the converse is not true. In fact, conditions (8), which are used to establish the usual stochastic ordering of skip-free-to-the-right CTMCs, are much more involved than conditions (5), which are used to establish the corresponding level crossing ordering. In order to have the CTMCs ordered in the usual sense, over and above the simple validity of the conditions (4) and (5) with $\alpha=1$, the sufficient conditions derived in Lemma 1 require an inequality between left-row sums for different rows of the generator matrices associated to the two CTMCs being compared.

In this respect, Irle and Gani [6, Theorem 4.1] showed that the ordering in distribution of the transition probabilities of two skip-free-to-the-right DTMCs (which can be viewed as SMPs with unitary times between consecutive transitions), for any common initial state, is sufficient to guarantee the corresponding level crossing ordering. Moreover, Irle [5, Theorem 2.1] established that the ordering in distribution of the transition probabilities, for any common initial state, along with the inverse ordering of the holding times between transitions, guarantees the level crossing ordering of skip-free-to-the-right SMPs.

We recall that a skip-free-to-the-right CTMC $X$, with state space $I$ and generator matrix $Q$, may be interpreted as an SMP that has holding times in state $i$ exponentially distributed with rate $q_{i}=\sum_{j \neq i} q_{i j}$, irrespective of the state visited at the next transition, and one-step embedded transition probability matrix $\boldsymbol{P}=\left(p_{i j}\right)_{i, j \in I}$ such that $p_{i j}=q_{i j} / q_{i}$ if $q_{i}>0$ and $p_{i j}=\delta_{i j}$ if $q_{i}=0$. By taking into account [5, Remark 2.2], the translation of [5, Theorem 2.1] for skip-free-to-the-right CTMCs leads to the following result.

Lemma 2. Let $X^{(1)}$ and $X^{(2)}$ be two lower-uniformizable skip-free-to-the-right CTMCs with common ordered state space I, order-isomorphic to some bounded or unbounded interval of $\mathbb{Z}$; have vectors $\boldsymbol{q}^{(1)}$ and $\boldsymbol{q}^{(2)}$ of transition rates from states, respectively; and have one-step embedded transition probability matrices $\boldsymbol{P}^{(1)}$ and $\boldsymbol{P}^{(2)}$, respectively. If

$$
q_{i}^{(1)} \leq q_{i}^{(2)} \text { and } \boldsymbol{P}_{i .}^{(1)} \leq_{\mathrm{st}} \boldsymbol{P}_{i .}^{(2)}, \quad \text { for all } i \in \bar{I},
$$

then $X^{(1)} \leq \mathrm{lc} X^{(2)}$.

We next present an example of two skip-free-to-the-right CTMCs that satisfy either conditions (3) or ((4), (5)) (sufficient for their level crossing ordering) but do not satisfy either $((7),(8))$ or $(9)$. The CTMCs in the example are Poisson shock models with exponential total repair (see, for example, [12]). That is, each of these processes is a CTMC, with state space $\mathbb{N}$, 
that counts the number of faults in a device subjected to unitary random shocks, occurring as a Poisson process with rate modulated by the state of the process. Each CTMC also has instantaneous exponential total repairs, with rate modulated by the state of the process; thus, its infinitesimal rates are of the form $q_{i j}=\lambda_{i} \delta_{j, i+1}+v_{i} \mathbf{1}_{\{j=0, i>0\}}$ for $i \neq j$, with $\lambda_{i}\left(v_{i}\right)$ denoting the shock (total repair) rate when the device has $i$ faults.

Example 1. For $r=1,2$, let $X^{(r)}$ be a Poisson shock model with exponential total repair, having shock rates $\lambda_{i}^{(r)}=\lambda^{(r)}$ and total repair rates $v_{i}^{(r)}=\lambda^{(r)}+\beta^{(r)} \mathbf{1}_{\{i \geq a\}}$, with $\beta^{(1)}, \beta^{(2)}>0$ and $a \geq 2$, such that

$$
\lambda^{(1)}<\lambda^{(2)}<\lambda^{(1)}+\frac{1}{2}\left(\beta^{(1)}-\beta^{(2)}\right)
$$

\section{Birth-death processes with catastrophes}

In this section, we apply the results obtained in the previous section to BDC processes (see [1], [2], and [3]) to derive sets of sufficient conditions for the level crossing ordering of such processes. We note that any skip-free-to-the-right CTMC on a subset of $\mathbb{N}$ may be interpreted as a BDC process describing the time evolution of the number of entities (individuals in a population, failures in a device, etc.).

Definition 3. Let $I$ be a subset of $\mathbb{N}$; let $\boldsymbol{\lambda}=\left(\lambda_{i}\right)_{i \in I}, \boldsymbol{\mu}=\left(\mu_{i}\right)_{i \in I}$, and $\boldsymbol{\beta}=\left(\beta_{i}\right)_{i \in I}$ be nonnegative vectors such that $\lambda_{\sup I}=0$ if $I$ is bounded above, $\mu_{\inf I}=0$, and $\beta_{\inf I}=0$; and let $\boldsymbol{C}=\left(c_{i j}\right)_{i, j \in I}$ be a lower-triangular stochastic matrix.

Then, a process $X=(X(t))_{t \in \mathbb{R}_{+}}$is a $(I, \boldsymbol{\lambda}, \boldsymbol{\mu}, \boldsymbol{\beta}, \boldsymbol{C})$ BDC process if it is a skip-free-to-theright CTMC with state space $I$ and generator matrix $\boldsymbol{Q}=\left(q_{i j}\right)_{i, j \in I}$, where

$$
q_{i j}=\beta_{i} c_{i j}+\mu_{i} \delta_{j, i-1}+\lambda_{i} \delta_{j, i+1}, \quad i \neq j .
$$

The usual interpretation of the parameters of a BDC process is as follows: $\lambda_{i}, \mu_{i}$, and $\beta_{i}$ are, respectively, the birth, death, and catastrophe rates of $X$ in state $i$. Moreover, $\boldsymbol{C}$ is the catastrophe probability matrix and $c_{i j}$ denotes the probability that state $j$ results from a catastrophe taking place in state $i$.

Important types of catastrophe families are described, for example, in [1] and [3]. These include $\operatorname{binomial}(p)$, where $0 \leq p \leq 1$; geometric $(p)$, where $0 \leq p \leq 1$; uniform; deterministic $(\boldsymbol{f})$, where $\boldsymbol{f}=\left(f_{i}\right)_{i \in I}$ is a vector such that $f_{i} \leq i$ for all $i \in I$; and total. Some details on the catastrophe probability matrices associated with each of these types of catastrophe families are given in Table 1. In the following, we do not distinguish between the type of catastrophe and the associated catastrophe probability matrix; thus we write, for

TABLE 1: Important types of catastrophe families. Here, $c_{i j}$ are the entries of the catastrophe probability matrix $C$.

\begin{tabular}{lcc}
\hline Type of catastrophe & $c_{i j}, 0 \leq j \leq i$ & $\underline{c}_{i k}, 0 \leq k \leq i$ \\
\hline $\operatorname{binomial}(p), p \in[0,1]$ & $\left(\begin{array}{c}i \\
j\end{array}\right) p^{i-j}(1-p)^{j}$ & $\sum_{j=0}^{k}\left(\begin{array}{l}i \\
j\end{array}\right) p^{i-j}(1-p)^{j}$ \\
geometric $(p), p \in[0,1]$ & $p^{i} \delta_{j 0}+(1-p) p^{i-j} \mathbf{1}_{\{j>0\}}$ & $p^{i-k}$ \\
uniform & $1 /(i+1)$ & $(k+1) /(i+1)$ \\
deterministic $(\boldsymbol{f}), 0 \leq f_{i} \leq i$ & $\delta_{j f_{i}}$ & $\mathbf{1}_{\left\{k \geq f_{i}\right\}}$ \\
total & $\delta_{j 0}$ & 1 \\
\hline
\end{tabular}


TABLE 2: Some ordering relations associated to catastrophe probability matrices.

\begin{tabular}{llcc}
\hline $\boldsymbol{C}^{(1)}$ & $\boldsymbol{C}^{(2)}$ & $\boldsymbol{C}_{i \cdot}^{(1)} \leq_{\mathrm{st}} \boldsymbol{C}_{i \cdot}^{(2)}$ & $\boldsymbol{C}^{(1)} \leq_{\mathrm{K}} \boldsymbol{C}^{(2)}$ \\
\hline $\operatorname{binomial}\left(p_{1}\right)$ & binomial$\left(p_{2}\right)$ & $p_{1} \geq p_{2}$ & $p_{1} \geq p_{2}$ \\
$\operatorname{geometric}\left(p_{1}\right)$ & geometric $\left(p_{2}\right)$ & $p_{1} \geq p_{2}$ & $p_{1} \geq p_{2}$ \\
$\operatorname{binomial}\left(p_{1}\right)$ & geometric $\left(p_{2}\right)$ & $p_{1} \geq p_{2}$ & $p_{1} \geq p_{2}$ \\
uniform & uniform & yes & yes \\
deterministic $\left(\boldsymbol{f}^{(1)}\right)$ & deterministic $\left(\boldsymbol{f}^{(2)}\right)$ & $\boldsymbol{f}^{(1)} \leq \boldsymbol{f}^{(2)}$ & $f_{i}^{(1)} \leq f_{m}^{(2)}, i \leq m$ \\
total & arbitrary & yes & yes \\
\hline
\end{tabular}

example, $\boldsymbol{C}=\operatorname{binomial}(p)$ whenever $\boldsymbol{C}$ is the catastrophe probability matrix of $\operatorname{binomial}(p)$ catastrophes.

We next apply Theorem 2 to compare BDC processes in level crossing. We recall that, with $I$ being a set of natural numbers, a stochastic matrix $\boldsymbol{A}=\left(a_{i k}\right)_{i, k \in I}$ is stochastically smaller, in the sense of Kalmykov order, than the stochastic matrix $\boldsymbol{B}=\left(b_{i k}\right)_{i, k \in I}$ if $\boldsymbol{A}_{i}$. $\leq_{\mathrm{st}} \boldsymbol{B}_{m}$. for all $i, m \in I$ such that $i \leq m$ (see, for example, [8, p. 148, Definition 3.16]). This is written as $\boldsymbol{A} \leq_{\mathrm{K}} \boldsymbol{B}$.

Theorem 3. Let I be a subset of $\mathbb{N}$, and let $X^{(r)}$ be a $\left(I, \lambda^{(r)}, \boldsymbol{\mu}^{(r)}, \boldsymbol{\beta}^{(r)}, C^{(r)}\right)$ BDC process for $r=1,2$. Then we obtain

(i) $X^{(1)} \leq_{1 \mathrm{c}} X^{(2)}$ provided that, for some $\alpha \in(0,1]$, the following conditions hold:

$$
\begin{aligned}
\lambda_{i}^{(1)} \leq \alpha \lambda_{i}^{(2)} \wedge \mu_{i}^{(1)} & \geq \alpha \mu_{i}^{(2)} \wedge \beta_{i}^{(1)} \geq \alpha \beta_{i}^{(2)} \quad \text { for all } i \in \bar{I}, \\
C_{i}^{(1)} & \leq{ }_{\mathrm{st}} \boldsymbol{C}_{i}^{(2)} \quad \text { for all } i \in \bar{I} ;
\end{aligned}
$$

(ii) $X^{(1)} \leq_{\mathrm{st}} X^{(2)}$ provided that the following conditions hold:

$$
\begin{gathered}
\lambda_{j}^{(1)} \leq \lambda_{j}^{(2)} \wedge \mu_{i}^{(1)} \geq \mu_{m}^{(2)} \wedge \beta_{i}^{(1)} \geq \beta_{m}^{(2)} \text { for all } j \text { and } i \leq m, \\
C^{(1)} \leq_{\mathrm{K}} \boldsymbol{C}^{(2)} .
\end{gathered}
$$

Proof. Let $X^{(1)}$ and $X^{(2)}$ be as stated. We note that, in view of (10), the conditions ((4), (5)), applied to $X^{(1)}$ and $X^{(2)}$, are equivalent to

$$
\lambda_{i}^{(1)} \leq \alpha \lambda_{i}^{(2)} \wedge \mu_{i}^{(1)} \delta_{i-1, k}+\beta_{i}^{(1)} \underline{c}_{i k}^{(1)} \geq \alpha\left(\mu_{i}^{(2)} \delta_{i-1, k}+\beta_{i}^{(2)} \underline{c}_{i k}^{(2)}\right)
$$

for all $i \in \bar{I}$ and $k<i$. Moreover, as

$$
\boldsymbol{C}_{i .}^{(1)} \leq_{\mathrm{st}} \boldsymbol{C}_{i .}^{(2)} \Leftrightarrow \underline{c}_{i k}^{(1)} \geq \underline{c}_{i k}^{(2)} \quad \text { for all } i \in \bar{I} \text { and all } k<i,
$$

we conclude that if there exists $\alpha \in(0,1]$ such that (11) and (12) hold, then (14) holds for all $i \in \bar{I}$ and $k<i$ and, in view of Theorem 2, it follows that $X^{(1)} \leq_{\mathrm{lc}} X^{(2)}$.

The second statement follows similarly, using the fact that $\boldsymbol{C}^{(1)} \leq_{\mathrm{K}} \boldsymbol{C}^{(2)}$ if and only if $\underline{c}_{i k}^{(1)} \geq \underline{c}_{m k}^{(2)}$ for all $i \leq m$ and all $k$.

Table 2 presents some situations where the ordering relations (12) and (13) involving catastrophe probability matrices hold, which are relevant for the use of Theorem 3. Note, in particular, that binomial and geometric catastrophes (see [1] and [3]) are stochastically 
decreasing in the parameter and total catastrophes (see [2]) are the smallest catastrophes, in both the usual and the Kalmykov senses.

Theorem 3(i) implies that BDC processes stochastically increase, in the sense of level crossing ordering, as the catastrophe distribution in each state increases stochastically in the usual sense. That is, BDC processes $X^{(1)}$ and $X^{(2)}$, which have common birth, death, and catastrophe rates but different catastrophe probability matrices $C^{(1)}$ and $C^{(2)}$, respectively, satisfy $X^{(1)} \leq_{\mathrm{lc}} X^{(2)}$ provided that $\boldsymbol{C}_{i}^{(1)} \leq_{\mathrm{st}} \boldsymbol{C}_{i}^{(2)}$ for all $i \in \bar{I}$. Thus, for example, BDC processes with binomial catastrophes stochastically decrease, in the sense of level crossing ordering, with the catastrophe probability.

Note also that if two BDC processes $X^{(1)}$ and $X^{(2)}$ have the same catastrophe probability matrix, it suffices to show that (11) holds in order to conclude that $X^{(1)} \leq_{\mathrm{lc}} X^{(2)}$. Let us illustrate the use of this result for birth-death (BD) processes. We note that a $(I, \boldsymbol{\lambda}, \boldsymbol{\mu}, \boldsymbol{\beta}, \boldsymbol{C})$ BDC process $X$ is a BD process if $\boldsymbol{\beta}$ is the null vector (this also makes the form of the catastrophe probability matrix $\boldsymbol{C}$ irrelevant), in which case we say that $X$ is a $(I, \boldsymbol{\lambda}, \boldsymbol{\mu})$ BD process.

Corollary 1. Let I be a subset of $\mathbb{N}$, and let $X^{(r)}$ be a $\left(I, \lambda^{(r)}, \mu^{(r)}\right)$ BD process for $r=1,2$. If

$$
\lambda_{i}^{(1)} \leq \alpha \lambda_{i}^{(2)} \wedge \mu_{i}^{(1)} \geq \alpha \mu_{i}^{(2)} \text { for all } i \in \bar{I} \text { and some } \alpha \in(0,1]
$$

then $X^{(1)} \leq_{\mathrm{lc}} X^{(2)}$. Moreover, if $X^{(1)}$ and $X^{(2)}$ are irreducible then the same conclusion is obtained if (15) is replaced by

$$
\sup _{i \neq \sup I} \frac{\lambda_{i}^{(1)}}{\lambda_{i}^{(2)}} \leq \alpha \leq \inf _{i \neq \inf I, i \neq \sup I} \frac{\mu_{i}^{(1)}}{\mu_{i}^{(2)}} .
$$

Note that Kirstein's conditions and Irle's conditions for irreducible BD processes (i.e. the appropriate translations of (7), (8), and (9)) are given by the following two sets of conditions:

$$
\begin{array}{r}
\lambda_{i}^{(1)} \leq \lambda_{i}^{(2)} \quad \text { and } \quad \mu_{i}^{(1)} \geq \mu_{i}^{(2)}, \quad i \in \bar{I}, \\
\lambda_{i}^{(1)}+\mu_{i}^{(1)} \leq \lambda_{i}^{(2)}+\mu_{i}^{(2)} \quad \text { and } \quad \frac{\mu_{i}^{(1)}}{\lambda_{i}^{(1)}} \geq \frac{\mu_{i}^{(2)}}{\lambda_{i}^{(2)}}, \quad i \in \bar{I} .
\end{array}
$$

Neither of these sets of conditions is equivalent to (16).

The number of customers in an $\mathrm{M} / \mathrm{M} / s / c$ system (see, for example, [4]) with arrival rate $\eta$ and death rate $\gamma$, where the system capacity $c$ may be either finite or infinite, may be viewed as a BD process on $\mathbb{N}$ with birth rates $\lambda_{i}=\eta \mathbf{1}_{\{0 \leq i \leq c-1\}}$ and death rates $\mu_{i}=\gamma \min (i, s)$. Therefore, we can easily derive the following application of Corollary 1 to $\mathrm{M} / \mathrm{M} / s / c$ systems.

Corollary 2. For $r=1,2$, let $X^{(r)}$ denote the number of customers in an $M / M / s^{(r)} / c^{(r)}$ system with arrival rate $\lambda^{(r)}$ and service rate $\mu^{(r)}$. If $c^{(1)} \leq c^{(2)}$ and

$$
\frac{\lambda^{(1)}}{\lambda^{(2)}} \leq \alpha \leq \frac{\mu^{(1)}}{\mu^{(2)}} \min \left(1, \frac{s^{(1)}}{s^{(2)}}\right)
$$

for some $\alpha \in(0,1]$, then $X^{(1)} \leq_{\mathrm{lc}} X^{(2)}$. 


\section{Acknowledgements}

This research was supported in part by Programa Operacional 'Ciência, Tecnologia, Inovação' (POCTI) of the Fundação para a Ciência e a Tecnologia (FCT), and co-financed by the European Community fund FEDER and the projects POSI/42069/CPS/2001, POSI/EIA/ 60061/2004, and POCTI/MAT/55796/2004.

\section{References}

[1] Brockwell, P. J., Gani, J. and Resnick, S. I. (1982). Birth, immigration and catastrophe processes. Adv. Appl. Prob. 14, 709-731.

[2] Chao, X. and Zheng, Y. (2003). Transient analysis of immigration birth-death processes with total catastrophes. Prob. Eng. Inf. Sci. 17, 83-106.

[3] Economou, A. And Fakinos, D. (2003). A continuous-time Markov chain under the influence of a regulating point process and applications in stochastic models with catastrophes. Europ. J. Operat. Res. 149, 625-640.

[4] Gross, D. AND Harris, C. (1998). Fundamentals of Queueing Theory, 3rd edn. John Wiley, New York.

[5] IrLe, A. (2003). Stochastic ordering for continuous-time processes. J. Appl. Prob. 40, 361-375.

[6] Irle, A. And Gani, J. (2001). Stochastic ordering and the detection of words. In Probability, Statistics and Seismology (J. Appl. Prob. Spec. Vol. 38A), ed. D. J. Daley, Applied Probability Trust, Sheffield, pp. 66-77.

[7] Kirstein, B. M. (1976). Monotonicity and comparability of time-homogeneous Markov processes with discrete state space. Math. Operationsforsch. Statist. 7, 151-168.

[8] Kulkarni, V. (1995). Modeling and Analysis of Stochastic Systems. Chapman and Hall, London.

[9] Müller, A. ANd Stoyan, D. (2002). Comparison Methods for Stochastic Models and Risks. John Wiley, Chichester.

[10] ReSNICK, S. (1992). Adventures in Stochastic Processes. Birkhäuser, Boston, MA.

[11] Shaked, M. And Shanthikumar, J. (1994). Stochastic Orders and Their Applications. Academic Press, San Diego, CA.

[12] Singh, H. And Jain, K. (1989). Preservation of some partial orderings under Poisson shock models. Adv. Appl. Prob. 21, 713-716. 